\title{
Discriminative compartmental CSs in shuttlebox avoidance acquisition
}

\author{
P. E. FREEDMAN and B. STEVEN CALLAHAN, University of \\ Illinois at Chicago Circle, Chicago, Illinois, 60680
}

Acquisition of shuttlebox avoidance by rats was recorded under four CS conditions: (1) light as CS always in one compartment, tone as CS always in the other (LTF), (2) 50\% light CS and 50\% tone CS but randomly occurring in either compartment (LTR); (3) light $C S$ in both compartments $(L L) ;(4)$ tone $C S$ in both compartments (TT). In per cent avoidance, $L L$ was superior to the other three groups, which did not differ from each other. The hypothesis that discriminably different stimuli used as CSs would reduce conflict and consequently facilitate avoidance acquisition was rejected. The factors which differentiate unidirectional and shuttle situations were discussed in light of these results.

Much work has been done comparing avoidance performance in unidirectional and shuttle situations. Performance in unidirectional situations has been typically characterized by rapid acquisition and high asymptotic levels of per cent avoidance, while avoidance responding in shuttle situations has usually shown relatively slower and less consistent acquisition with lower asymptotes. One of the hypotheses for this relative decrement in performance in shuttle situations has been termed "the staying hypothesis" by Theios (1966). In effect, this hypothesis is based on the fact that, in the shuttle situation, the organism has to return to a place where it has just previously been shocked. This sets up an approach-avoidance conflict which provides for a response that competes with the required active avoidance response. This competing response is a passive avoidance response to the other compartment. Thus, due to the conflict brought about by the competing "staying response," poor avoidance behavior results. In the unidirectional situation, the organism is not required to return to a place where it has been previously shocked. There is only one "safe" and one shock compartment used for the same purpose throughout the entire experiment. Therefore, the relative decrement in avoidance responding found in shuttle situations does not occur.

Handling is one additional variable which has been cited as a differential factor. The work of Wahlsten, Sharp, \& Cole (1967) demonstrated that handling did have a differential effect on avoidance responding in comparing situations of the unidirectional and shuttle types. But it was concluded that handling had only a weak effect on freezing and that the immediate aftereffects of it were not the critical aspects which facilitated avoidance in unidirectional situations.

The present experiment explored the problem of what cues the animal attends to in making an avoidance response, and perhaps developing the competing behaviors already mentioned. A shuttling situation was used where one CS was always presented for a response from left to right and another CS was always. presented for a response from right to left. It was hypothesized that, after some conditioning, the onset of the CS in Compartment $A$ would lead to the discrimination that Compartment $B$ was "safe." Conversely, the onset of the CS in Compartment B would then indicate to $S$ that Compartment A was "safe." Therefore, it was expected that $S$ would respond as if the shuttling task was two one-way avoidance situations with two different CSs, in this case a light and a tone. Since, in this type of shuttling situation, the $S$ must learn the avoidance response to two different stimuli and in effect, learn two different avoidance responses at once, the typical shuttling situation using the same CS in both compartments was thought to be an inadequate control. Therefore, a control group was run such that the light and tone were randomly presented as CSs $50 \%$ of the time in each compartment. Groups with light or tone presented $100 \%$ of the time in both compartments were also run to test the relative efficacy of each of these two CSs.

\section{SUBJECTS}

The Ss were 56 male albino Holtzman rats. They were maintained on ad lib food and water in group cages one week prior to and throughout the study and were 90 days old when received. APPARATUS

The shuttlebox was a $24 \times 8$ in. box with a ceiling height of $10-1 / 4$ in. The box had an unpainted backwall and ceiling, sheetmetal end walls, a clear Plexiglas front and a stainless-steel grid floor. The box was divided into two equal compartments by a sheetmetal wall which had a 4-1/2 in. high and 4 in. wide opening at floor level. No door was used. Shock could be applied to the end-wall, grid and sheetmetal divider for each compartment independently. The shock was a constant current source of $.3 \mathrm{~mA}$. A $7-1 / 2 \mathrm{~W}$ light and a $4 \mathrm{in}$. speaker were mounted over the ceiling of each compartment, the light being 2 in. and the speaker 6-5/8 in. from the back wall. The speaker delivered a $2000 \mathrm{cps}$ tone at $80 \mathrm{~dB}$ measured at the grid floor. Red-filtered photocell beams mounted 3-1/2 in. from each side of the divider at a height of $1-7 / 8$ in. from the grid floor in each compartment provided a record of S's response.

The entire system was automatically controlled by a solid state program and avoidances were recorded on a printout counter. The shuttlebox was in a dark room separated from the programming equipment.

\section{PROCEDURE}

Fourteen Ss were randomly assigned to each of four groups. The groups were distinguished by the CS condition received. Group Light-Tone-Fixed (LTF) received light as a CS in one compartment and tone as a CS in the alternate compartment. Half of the Ss in this group had light on the right side and tone on the left. This was reversed for the other half of the Ss. Group Light-Tone-Random (LTR) received $50 \%$ of their trials to a light CS and 50\% to a tone CS, but the light or tone was presented as the CS in either compartment on a random $50 \%$ schedule-as determined by a random probability unit. Group Tone-Tone (TT) had tone as a CS on every trial and Group Light-Light (LL) had light as a CS on every trial.

On the first day of the experiment, $S$ was allowed $10 \mathrm{~min}$ exploratory time in the apparatus with all stimuli off. He then received his first trial. The CS came on $5 \mathrm{sec}$ prior to the onset of the shock, and the shock and/or the CS were response terminated. The intertrial interval was 30 sec. Each $S$ received 40 trials per day for 10 days. Intertrial responding was controlled by applying shock to the alternate grid. During the CS-shock interval there was no shock in either compartment. If $S$ avoided, shock was initiated in the vacated compartment $.5 \mathrm{sec}$ after the response.

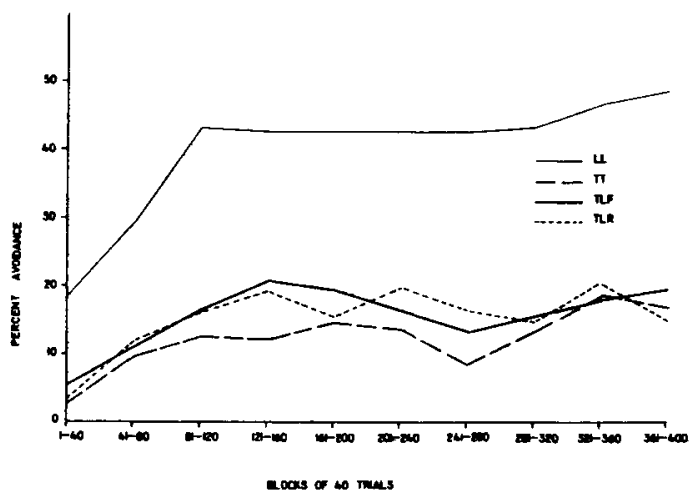

Fig. 1. Per cent avoidance for LL, TT, TLF, and TLR over blocks of 40 trials (days). 


\section{RESULTS}

The results are shown in Fig. 1 in terms of mean per cent avoidance per day over 10 days. A mixed design analysis of variance with $C S$ condition as the between effect and with trial blocks (TB) and the TB and CS interaction as within effects indicated that $\mathrm{CS}(\mathrm{F}=9.79 . \mathrm{df}=3 / 52, \mathrm{p}<.001)$ and trial blocks $(\mathrm{F}=13.51, \mathrm{df}=7 / 468, \mathrm{p}<.001)$ were significant variables. $A$ stringent Tukey Test applied to the simple CS effects indicated that LL avoidance was significantly superior to TT, LTF, LTR $(p<.05)$ which were not different from one another.

\section{DISCUSSION}

The finding that the LTF and LTR groups demonstrated almost identical avoidance performance seems to negate the hypothesis that conflict behavior will be reduced in a shuttling situation if one CS is used to indicate a response in one direction and another CS is used to indicate the response in the opposite direction. It, therefore, seems that $S$ does not attend directly to the CS as a discriminant cue in making an avoidance response. Since there is evidence that $S$ could discriminate between compartments, it is important to consider what other cues might be critical to the acquisition of conflict behaviors.

That the animals could discriminate between compartments was apparent from the data in the current study. Ss were always placed in and removed from the right compartment at the beginning and end, respectively, of each daily session. Consequently, they spent more "safe" time on the right. Collapsing across groups and trials, it was found that 2,909 avoidance responses were made into the right side, while only 1,711 avoidance responses were made into the left side. This ratio was reflected in all groups. Therefore, the direction of the response and/or the relative spatial location of the compartment appear to be discriminable and potentially critical cues that the animal responds to in the avoidance situation. It is also possible here that $S$ was responding to the "safe" compartment rather than to the "safe" direction or spatial location.

The importance of spatial location as a critical cue is supported by Olton \& Issacson (1968). In their study they compared avoidance acquisition in unidirectional and shuttling situations to that of a situation utilizing the McCleary technique. The McCleary technique is unidirectional with respect to spatial and directional cues, but bidirectional with respect to compartment cues. Olton and Isaacson's results show that the performance of animals in the McCleary situation was significantly better than that of animals in a typical bidirectional shuttling situation and very close to the performance of animals run under standard unidirectional conditions. It was then concluded that rats rely more on spatial than visual cues while performing an active avoidance task and that they learn this task by associating the noxious stimulation with a particular spatial location or direction. To this conclusion the present study suggests, in addition. a minimal role for discrimination of CS cues in the conflict interpretation of shuttlebox performance.

Research by Wedeking (1967) has also emphasized response factors involved in shuttlebox avoidance. Wedeking demonstrated rapid acquisition and high asymptotic performance with a dual. one-way shuttle apparatus. Here $S$ s had a different passage to go from $A$ to $B$ than they had for going from $B$ to $A$. Therefore, the kinesthetic cues of the response from $A$ to $B$ and from $B$ to $A$ were discriminable.

An additional comment should be made concerning the marked superiority of the LL groups in the present study, suggesting the possibility that the $7-1 / 2 \mathrm{~W}$ light was initially noxious. To test this hypothesis, 10 naive Ss were given 30 test trials on each of two days. A trial consisted of turning on the light in the occupied chamber for $20 \mathrm{sec}$ or until $\mathrm{S}$ left the chamber. This was followed by a $20 \mathrm{sec}$ period of no light in which the S's shuttle behavior was observed. No shock was ever presented. A total of 267 shuttle responses were made from the lighted compartment. However, Ss shuttled at least once in each of 278 of the non-light intervals. In addition, there was no indication of an increase in shuttling or a decrease in latency over trials. Consequently, the hypothesis that the light was initially noxious was rejected. Two other hypotheses can be suggested to explain the superiority of the LL group over the TT group: (a) light stimuli acquire noxious properties more rapidly than tone stimuli when paired with shock; or (b) the spatial location of the light-being nearer the end wall of the compartment-and the spatial properties of light stimuli are more commensurate with an avoidance response which requires moving away from the source of stimulation. The latter interpretation would be supportive of the directional variable in avoidance acquisition. At present, research is underway to differentiate these two hypotheses.

\section{REFERENCES}

OLTON, DAVIS S., \& ISAACSON, ROBERT L. Importance of spatial location in active avoidance tasks. Journal of Comparative \& Physiological Psychology, 1968, 65, 535-539.

THEIOS, J., LYNCH, A. D., \& LOWE, W. F. Differential effects of shock intensity on one-way and shuttle avoidance conditioning. Journal of Experimental Psychology, 1966, 72, 294-299.

WAHLSTEN, D., SHARP, D., \& COLE, M. Improvement of shuttle avoidance by handling during the intertrial interval. Psychonomic Society, 1967.

WEDEKING, PAUL W. Rat avoidance behavior in a dual, one-way shuttle apparatus. Psychonomic Science, 1967, 8, 33-34. 